\title{
Folding and stretching a thermoelectric generator
}

Jhonathan Prieto Rojas, Mutee Ur Rehman, Mohammed Aieash Albettar, David Conchouso, Arpys Arevalo, et al.

Jhonathan Prieto Rojas, Mutee Ur Rehman, Mohammed Aieash Albettar, David Conchouso, Arpys Arevalo, Devendra Singh, lan Foulds, Muhammad M. Hussain, "Folding and stretching a thermoelectric generator," Proc. SPIE 10639, Micro- and Nanotechnology Sensors, Systems, and Applications X, 106390E (8 May 2018); doi: 10.1117/12.2304992

SPIE. Event: SPIE Defense + Security, 2018, Orlando, Florida, United States 


\title{
Folding and Stretching a Thermoelectric Generator
}

\author{
Jhonathan Prieto Rojas ${ }^{* a}$, Mutee Ur Rehman ${ }^{\mathrm{a}}$, Mohammed Aieash Albettar ${ }^{\mathrm{a}}$, David Conchouso ${ }^{\mathrm{b}}$, \\ Arpys Arevalo ${ }^{\mathrm{b}}$, Devendra Singh ${ }^{\mathrm{b}}$, Ian Foulds ${ }^{\mathrm{c}}$, Muhammad M. Hussain ${ }^{\mathrm{b}}$. \\ ${ }^{a}$ Electrical Engineering Department, King Fahd University of Petroleum and Minerals (KFUPM), \\ Dhahran 31261, Saudi Arabia. \\ ${ }^{\mathrm{b}}$ Computer, Electrical and Mathematical Sciences \& Engineering Division, King Abdullah Univ. of \\ Science and Technology (KAUST), Thuwal 23955, Saudi Arabia. \\ ${ }^{\mathrm{c}}$ School of Engineering, The University of British Columbia, Okanagan Campus, Kelowna, British \\ Columbia, Canada V1V1V7.
}

\begin{abstract}
As we are at the verge of entering the era of Internet-of-Things (IoT), there is a clear need to produce continuous power supply to the huge amount of electronic devices that must be wirelessly interconnected and operate uninterruptedly. At the same time, new mechanical constrains arise from the fact that these devices should be ubiquitous, which leads to the need of lightweight and mechanical compliance to any shape or surface. As an important renewable energy source, a mechanically adaptable thermoelectric generator (TEG) can make use of the usually wasted thermal differences between ambient and technology-users to power-up such systems. With this idea in mind, we have developed a simple approach to fabricate TEGs, based on commonly available substrates (paper or polymers) and assisted through simple folding and cutting techniques (born from origami and kirigami) to form strategic structures (serpentine, helical, spiral, etc.) with the mechanical advantage of foldability and over $100 \%$ demonstrated stretchability. The use of these methods and structures allows the mechanical reconfigurability of the devices to, for example, increase the temperature difference in a TEG, thus its power, or allow a more efficient use of area and therefore increase the power density. We will discuss the strategies to effectively integrate folding and cutting techniques with common materials and the basic TEG configuration, as well as demonstrate the devices' implementation and characterization. Finally, we believe our simple integration approach offers an interesting and versatile methodology, which can be easily extrapolated to new materials and technologies for a greater variety of applications.
\end{abstract}

Keywords: Thermoelectric generators, flexible electronics, stretchable electronics, origami, kirigami, paper, wearable technologies, mechanical reconfigurability.

\section{INTRODUCTION}

Recent advancements in electronics, materials, and development of new fabrication techniques have opened the doors to a new era for electronics devices. These achievements have resulted into many innovative technologies and devices such as wearable electronics, bio-implantable devices, soft robotics and Internet-of-things (IoT), etc ${ }^{1-8}$. Nevertheless, these devices encounter many mechanical constraints as they are to be mounted on irregular geometries and surfaces. This give rise to another very important class of electronics devices, that are conformal and can bend, flex as well as stretch according to the application, while maintaining their conventional electrical performance ${ }^{9-11}$. This new class of electronics has attracted very much attention over the past few decades and have seen tremendous growth. The ability of these flexible and stretchable devices to be conformal to complex geometries have given them the advantage over the conventional brittle and rigid electronics. To have the best of these devices, several techniques are being explored, including taking naturally flexible, insulating materials, like many polymers, and making them electrically conductive ${ }^{12-}$ ${ }^{14}$ or by taking conventional electronic materials and making them flexible and stretchable ${ }^{15-20}$. The second approach employs the fact that ultra-thin materials become less rigid and thus flexible. Moreover, stretchability can be achieved through structural modifications or using specific geometrical shapes, such as serpentine, spiral, helix and others ${ }^{16-18,21-}$ ${ }^{24}$. Furthermore, very efficient fabrication techniques have made it easier to fabricate these devices with complex geometrical structures for better stretchability and mechanical performance under strain ${ }^{20}$.

*iprojas@kfupm.edu.sa; phone 96613860 8867; fax 966138603535

Micro- and Nanotechnology Sensors, Systems, and Applications X, edited by Thomas George,

Achyut K. Dutta, M. Saif Islam, Proc. of SPIE Vol. 10639, 106390E · @ 2018 SPIE

CCC code: $0277-786 \mathrm{X} / 18 / \$ 18 \cdot$ doi: $10.1117 / 12.2304992$ 
In addition to efficient fabrication techniques and novel materials, these flexible devices must be lightweight, durable, and most importantly energy efficient, so that mobility and wearability can be truly achieved. Likewise, finding efficient ways to power up such electronic devices for uninterrupted operation is also a challenge. Therefore, efforts are underway to devise new ways to develop power sources with same mechanical properties as the devices themselves, such as flexibility and even stretchability. Hence, development of a stretchable and flexible energy harvester might become the solution to this very important issue ${ }^{25}$.

Harvesting energy from unconventional sources is becoming more and more popular as it might provide cheaper and environmental friendly power alternatives. Among common power sources, thermal energy has been harvested to power up electronic devices, in our case the stretchable and flexible devices ${ }^{26-29}$. Several efforts and techniques, ranging from system optimization to new materials, are being engineered to harvest this readily available energy, and mostly wasted, for useful purposes ${ }^{28-30}$. Moreover, applications where electronic devices are to be mounted on human skin, such as wearable electronics, can harvest energy from human heat for their operations ${ }^{31-34}$. Thermoelectric generators (TEGs) are capable of harvesting energy through a temperature gradient, thus providing an environment friendly power source by harvesting this wasted energy ${ }^{29,30,35}$. A larger temperature difference between the hot and cold ends of a TEG result in higher output power ${ }^{25}$. Consequently, the development of a fully stretchable and foldable TEG, would allow us to produce adaptive output power by controlling the distance between the hot and cold ends, thus the temperature difference itself as shown in Figure $1^{25}$.

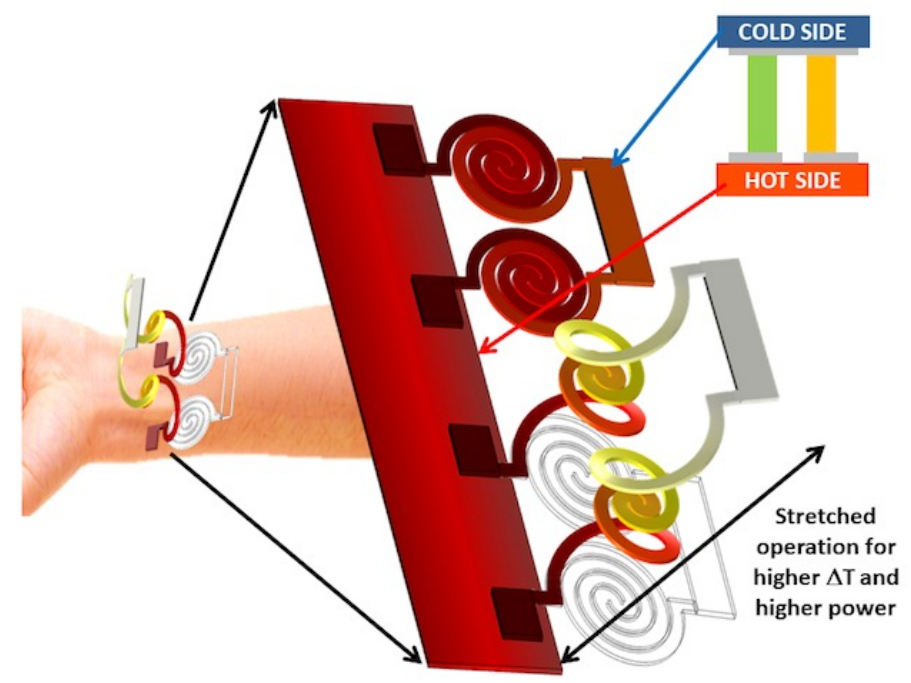

Figure 1. Stretchable TEG concept, illustrating the advantage of adaptively increasing the distance between cold and hot sides to increase power generation.

Organic materials might be the first choice when it comes to stretchable and flexible devices and researchers have explored these materials, as well as their nanocomposites. Several polymeric and organic materials have been used as flexible substrate for TEGs ${ }^{36-38}$. However, their thermal properties are much lower than many inorganic materials ${ }^{39}$. Since, most inorganic materials are rigid and brittle; therefore, a TEG composed of both organic and inorganic materials might produce better performance with the needed mechanical adaptability. Accordingly, polymers can be integrated with several inorganic materials that might have better electrical and thermal characteristics, thus their use could be more favorable ${ }^{40}$. These hybrid TEGs exploit the excellent mechanical characteristics of organic/polymeric materials, usually used as substrates, with the excellent thermoelectric properties of inorganic material. The organic material will act as a flexible substrate on top of which the inorganic material with excellent thermoelectric properties can be integrated/deposited. For example, polymers like polyimide (PI) and SU8 photoresist support advanced photolithographic techniques and thus interesting geometries can be designed with higher resolution ${ }^{41-43}$. Alternatively, paper has been also considered as a noteworthy structural material to develop foldable TEGs ${ }^{44}$. Additional to its affordability, adaptability and practicality, paper allows us to implement millenary techniques, such as origami and kirigami, which allow a more efficient use of area and other structural advantages ${ }^{45-47}$. For instance, either with the use 
of paper or a thin polymer, geometrical modifications can be introduced to any of these substrates; through cutting and folding, stretchable structures can be formed (serpentine, spiral, helix, etc). In consequence, stretchable and flexible TEGs can be thus developed, which integrate easily with flexible, as well as stretchable electronics, or can even exhibit enhanced power production ${ }^{40,48}$.

As mentioned above, geometrical modifications allow any kind of material, even rigid and brittle ones, to be transformed into stretchable platforms ${ }^{49}$. For example, spirals and serpentines are naturally stretchable geometries and shaping a thin film of almost any material with such geometries, results into not only flexible but also stretchable structures ${ }^{20,23,24}$. Double arm spirals are of especial interest, due to their higher achievable stretch ratio ${ }^{50,51}$ Further improvement can be achieved if compound structures are used, so that serpentines and spirals are combined together to reduce induced stress and strain, in a similar way as fractal structures work ${ }^{23}$.

In this paper, we describe the fabrication and integration of hybrid TEGs, where common substrates, such as paper and polymers, are used for mechanical support. The design and characterization of these flexible and stretchable energy harvesters are also explained, including the description of compound structures for increased mechanical performance. Such developments represent important steps towards truly mobile and wearable technologies with self-powering capabilities.

\section{FABRICATION}

\subsection{Materials and Designs}

We employed commonly available materials as substrate with different designs to evaluate the final TEG's performance. The TEG devices were based on polymeric or paper based substrates, integrated with well-known thermoelectric (TE) materials, bismuth telluride $\left(\mathrm{Bi}_{2} \mathrm{Te}_{3}\right)$ and antimony telluride $\left(\mathrm{Sb}_{2} \mathrm{Te}_{3}\right)$, sequentially deposited on the substrate to form TE pairs. The first design was based on off-stoichiometry thiol-enes (OSTE) polymer as substrate, shaped with six spiral structures per leg, connected in series for extended elongation. OSTE exhibits tunable mechanical properties although it is more complex to work with and no that common. The second implementation consisted of a simpler single-spiral-perleg design, for which we chose PI, a much more common polymer, as substrate along with the same TE materials. A simpler fabrication process was achieved with a much lower resistance. Furthermore, the performance of this second implementation was compared with polyester-paper material as substrate and with the same TE materials ${ }^{25}$. It is important to highlight that all these implementations were stretchable thanks to the helix/spiral structures. Additionally, a third and simpler implementation consisted of a paper based foldable TEG. These designs consisted of simple strips of TE materials deposited on top of the paper. Standard fiber-based paper and polyester-based paper were used as substrate, also for comparison purposes ${ }^{44}$.

\subsection{Stretchable TEGS}

The first design towards the structurally stretchable TEG employs the Off-Stoichiometry Thiol-Enes (OSTE) polymer. The fabrication process is rather complex, involving a double molding fabrication process. At first the desired spiral structure was designed in PMMA using a $\mathrm{CO}_{2}$ laser cutter. Next, PDMS based mold was casted in by its application on the PMMA spirals, which were glued to a PMMA-slab to form a hard mold, to act as a flexible mold for OSTE. After pouring the OSTE into the mold, UV cross linker was used to crosslink the structures. The released spirals, from the soft mold, were sequentially sputtered with the TE materials. Finally, the $\mathrm{Au} / \mathrm{Ti}$ was sputtered as interconnect between the TE materials ${ }^{25}$.

Due to the large length of each TE leg, the internal resistance ended up being too high. Additionally, the complex fabrication process involved in the OSTE processing made us look for alternatives, focusing on simpler approaches and materials that are more accommodating. Two possible alternatives, with less complicated fabrication processes involved, were found to be PI and polyester-paper. Polyester paper was chosen based on its smoother surface, temperature stability and stiffness compared to the standard fiber-based paper. The fabrication process for both of these materials is simpler and started with patterning the helical/spiral structure on these two substrates using the $\mathrm{CO} 2$ laser cutter. Same materials were used to cut their shadow masks through laser cutter. These masks were employed for the deposition of the TE materials to the thickness of $\sim 2 \mu \mathrm{m}$. To reduce the internal electrical resistance, both sides (top and down) of the helix/spirals were sputtered with the TE materials ${ }^{25}$. 


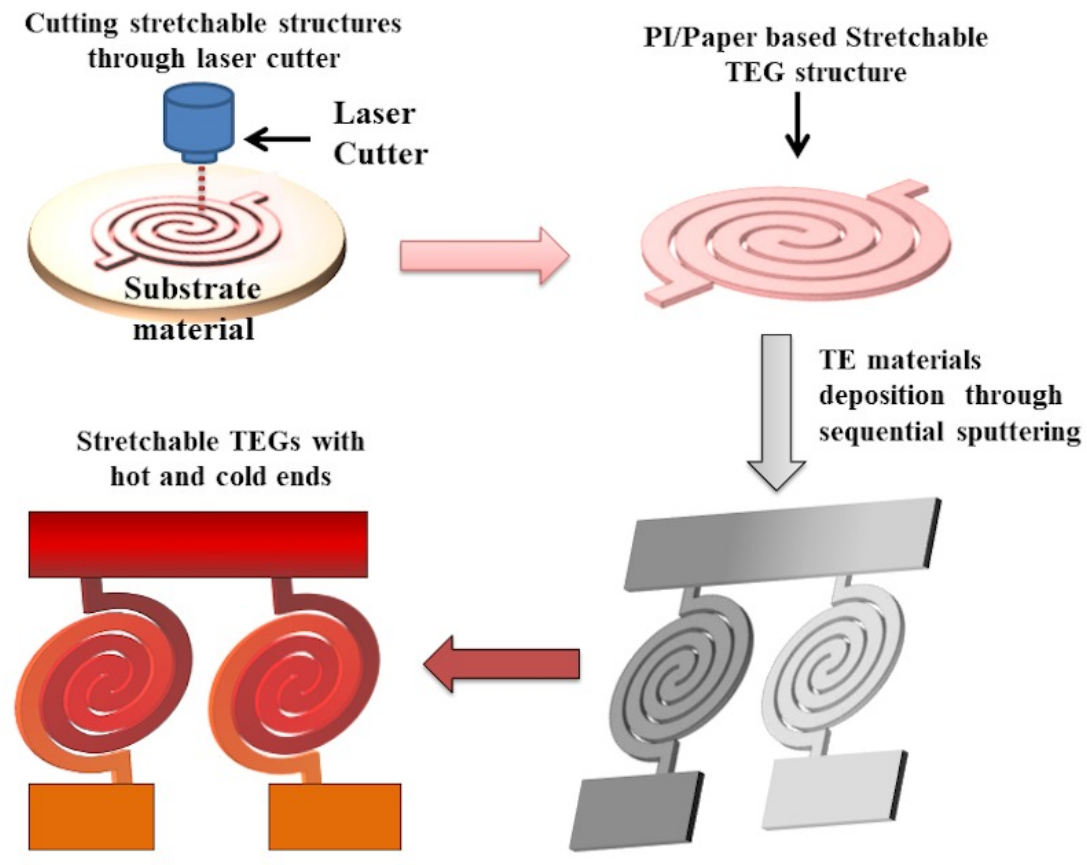

Figure 2. Fabrication process flow for implementing a single spiral TEG design, with either PI or paper-based substrates.

\subsection{Paper Based Foldable TEGs}

For the paper based TEGs two types of papers were used as substrates. One of them was a standard fiber-based paper and the other one was smoother, heat resistant polyester-based paper. Well-known TE materials, bismuth telluride $\left(\mathrm{Bi}_{2} \mathrm{Te}_{3}\right)$ and antimony telluride $\left(\mathrm{Sb}_{2} \mathrm{Te}_{3}\right)$, were used as active TE materials once again.

Initially, for the standard paper as substrate, four thermopiles of $\mathrm{Bi}_{2} \mathrm{Te}_{3}-\mathrm{Sb}_{2} \mathrm{Te}_{3}$ were deposited through a simple hardmask approach. The fabrication process involved the sputtering of $4 \mathrm{~cm} \times 2 \mathrm{~mm}$ TE material strips (through the hard mask) to a final thickness of $750 \mathrm{~nm}$. Gold strips were deposited to connect the TE pairs. The final dimensions of the TEG with paper substrate was of $4 \mathrm{~cm} \times 2 \mathrm{~cm}$, with foldable capabilities. In this case, by folding the paper substrate, thus changing the TEG's length, a higher temperature difference could be achieved, and additionally, it allows attachment to constantly moving hot and cold sides as illustrated in Figure $3^{44}$.

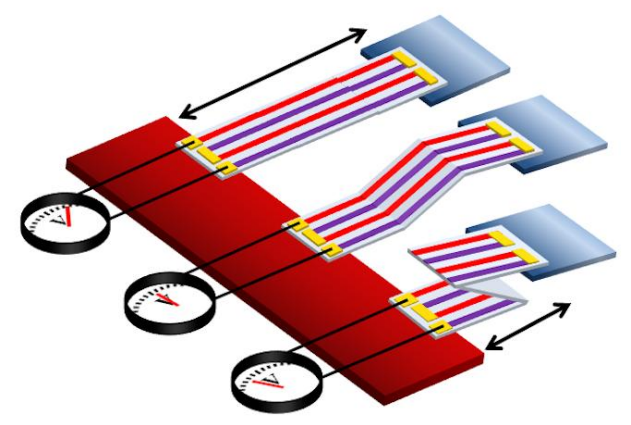

Figure 3. Foldable paper-based TEG with adaptive length, attached to mobile hot and cold sides.

A second paper-based design involved a polyester-based paper $(4 \mathrm{~cm} \mathrm{x} 1 \mathrm{~cm})$ as substrate with more pairs of the TE materials. Strips of the TE material were thicker and shorter ( $8 \mathrm{~mm}$ long and $\sim 2 \mathrm{um}$ thick), again to reduce the internal 
resistance of the overall structure compared to the previous design. Two devices were tested with the same area, one with $10 \mathrm{TE}$ pairs and other with $20 \mathrm{TE}$ pairs. The fabrication process involved an improved mask design on vinyl sticker using the $\mathrm{CO}_{2}$ engraving laser. Since, the mask was in direct contact with the paper substrate due to the adhesiveness of the materials, sequential deposition of the TE materials was observed to be finer and more accurate compared to the previous design. Moreover, due to the smoother surface of the polyester paper, the deposited TE materials appeared to be more uniform. In addition, the TE materials were directly connected to each other and the gold interconnects was not deposited for simplicity of the fabrication process ${ }^{44}$.

\subsection{Compound Structures to Enhance Stretchability}

As explained earlier, structural modifications not only could improve device performance, as in the case of stretchable TEGs, but also improve the mechanical stability of the device under stress. One of these strategies consist of arranging the brittle semiconducting materials, containing the active electronics, in arrays of rigid islands as shown in Figure 4 . In this arrangement, rigid islands, hosting the electronic components, are connected to each other through stretchable interconnects. The design of these interconnects is prepared in a way that the structure is able to mitigate strain induced during the flexing, bending, stretching or even twisting. The main idea behind this arrangement is to minimize the stress localization at the brittle components of the electronics ${ }^{12}$. Another benefit of this scheme is that it provides the freedom to separate and reorganize the different components of the system such as power management, sensor modules, communication, etc., as shown in Figure 4. Unlike the islands, these interconnects can be stretched due to their structure. Figure 4 also shows the spiral based interconnects, where a single spiral could stretchable to $\sim 1000 \%{ }^{20}$. In order to improve the mechanical performance of these stretchable interconnects, the straight arms of the spirals were replaced with a fractal inspired compound design that integrates both serpentine and spiral geometries. In addition, horseshoe structures were added at the beginning and at the end of the arms of spiral to further improve those points of higher stress accumulation. The overall design improved the mechanical performance of the structures, where the maximum stress was found to be approximately $50 \%$ less than the one observed in the spirals with the straight arms ${ }^{23}$. This strategy can be then applied to other stretchable technologies, such as stretchable TEGs, with the aim of optimizing the overall mechanical robustness.

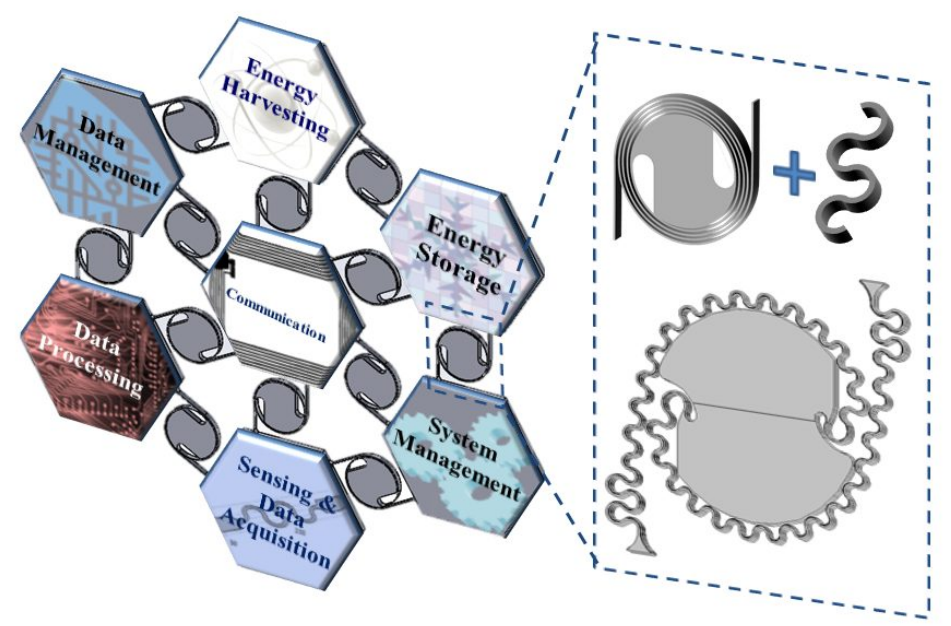

Figure 4. Schematic of a stretchable electronic system incorporating compound serpentine-spiral structures with optimized mechanical behavior.

\section{RESULTS AND CHARACTERIZATION}

In order to characterize the different implementation, the temperature was adjusted using a hot plate and a multimeter was used to measure the electrical parameters of TEGs. For the first OST-based TEG, the measurement results showed that the voltage change with temperature change increased upon stretching by about $40 \%$ due to the larger separation 
between the cold and hot ends of the TEG. On the other hand, it was also observed that the electrical resistance of the device also increased upon stretching at $100 \%$ elongation by near $25 \%$, thus limiting the current flow and consequently the power generation. The complex fabrication process and large internal resistance of the OSTE-based TEG motivated us to improve the design. The alternate design based on PI and polyester based paper was simple in fabrication and resulted in lower resistance. The resistance, in case of PI based design, was found to be $\sim 18 \mathrm{k} \Omega$ ( 2 order of magnitude less than the OSTE-based design) and upon stretching at $140 \%$ elongation, it increased by approximately double. Similarly, the resistance in case of paper-based design was observed to be higher than with the PI ( $42 \mathrm{k} \Omega)$, but it increased only about $15 \%$ after $60 \%$ elongation. Both of these designs are much simpler to fabricate and also present a lower electrical resistance. As it is also evident from the values of the resistance, PI based design resulted in higher resistance under strain compared to paper-based designs. This behavior could be accounted to the irregular surface of the paper compared to PI, which resulted in better stress distribution at elongation, thus having less impact on the electrical conductivity. Furthermore, the polyester paper-based TEG generated almost twice the power when stretched to $\sim 60 \%$ elongation, due to higher temperature difference as previously explained. An additional interesting observation was that PI-based TEG produced less power in the stretched state, compared to the un-stretched state, because of its higher thermal conductivity, even though the films were more electrically conductive than the polyester paper-based TEG ${ }^{25}$. Figure 5 shows our different designs at stretched state.

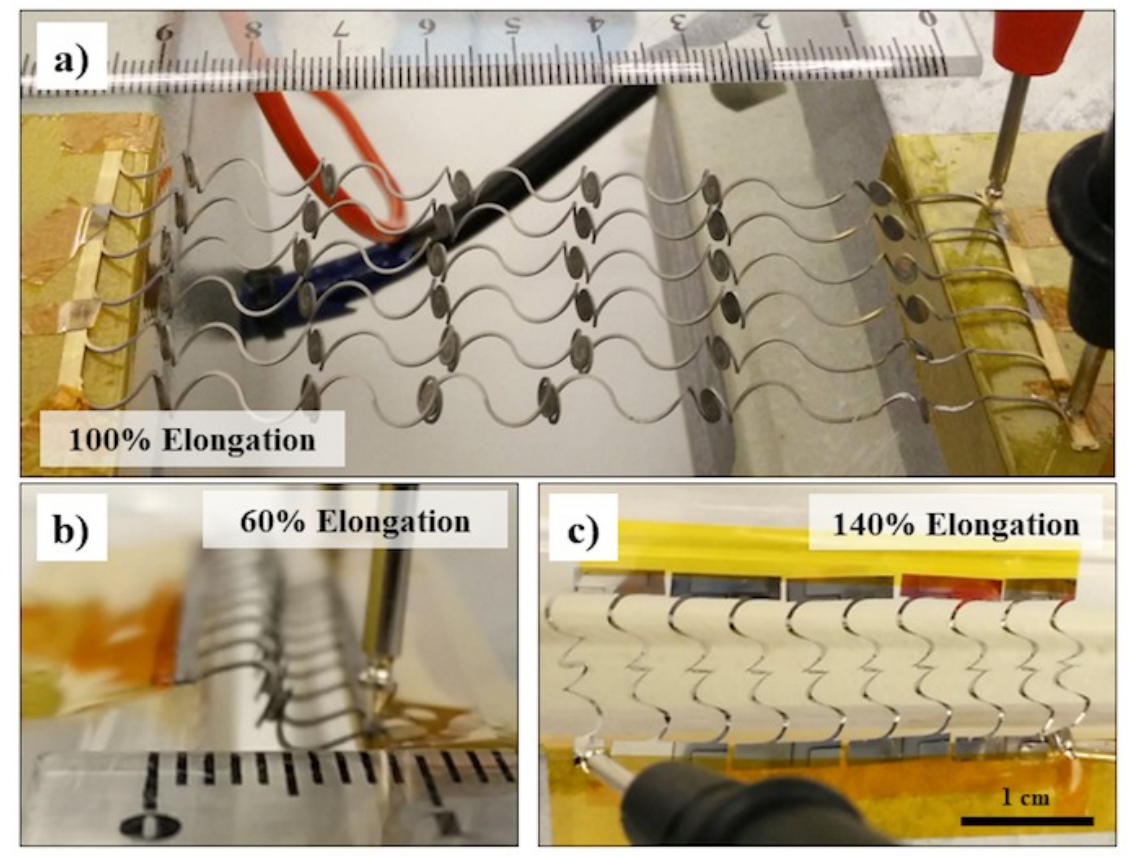

Figure 5. Stretchable TEG devices at different elongations, implemented with different materials and designs. a) OSTE-based multi-spirals TEG, b) Paper-based single-spiral TEG and c) PI-based single-spiral TEG.

In order to understand the mechanical behavior of these designs, they were simulated using finite element analysis (FEA) based on COMSOL software. The designs, drawn in CorelDraw, were imported to COMSOL for simulation. Geometric nonlinearities were also used in the simulation to account for large deformations. The design was stretched by moving the structure to a maximum of $100 \%$ elongation. PI and paper were used as the structural materials and $\mathrm{Bi}_{2} \mathrm{Te}_{3}(1$ $\mu \mathrm{m}$ thick) was used as the TE material deposited on these substrates. Simulation results for both designs showed that the maximum stress was found at the ends of the spirals. The maximum stress values were found to be far lower than ultimate tensile strengths for both the materials, when reaching maximum elongation to $100 \%$. Moreover, the maximum strain and stress values were observed to be at the points of maximum deformation for the $\mathrm{Bi}_{2} \mathrm{Te}_{3}$ film, thus contributing to the observed film's resistance increment ${ }^{25}$.

For paper-based foldable TEGs, the initial maximum power obtained, in case of standard paper, was too low (in the order of hundreds of $\mathrm{pW}$ at $50^{\circ} \mathrm{K}$ ). Such low performance can be attributed to very high internal resistance (in the order of $M \Omega$ ). This very high value of resistance is observed due to the irregular surface of the standard fiber-based paper. 
Next, two new designs on the standard fiber-based paper, with optimized 10 and 20 TE pairs were prepared and their electrical performance was studied. Higher power generation (by two orders of magnitude - to tens of nW) and lower resistance was observed (by one order of magnitude). It was also observed that the increase in power is not necessarily proportional to the number of TE pairs because of the resistance being increased due to the series connection. Finally, the same designs with optimized 10 and 20 TE pairs were replicated on a polyester-paper substrate and have shown even higher increase in power (more than 3 times higher than the fiber-based paper TEGs). This increase was attributed to the smoother surface and better paper's quality, at the cost of higher mechanical rigidity ${ }^{44}$.

\section{CONCLUSION}

We have developed several thermoelectric devices using different fabrication techniques and diverse stretchable/foldable designs, featuring the integration between inorganic and organic materials. Different materials have been tested as structural substrates and their effect on the overall performance of the device has been studied. The fabricated devices had the ability to stretch and bend, depending on the design, thus can be implemented for variety of applications. In the case of OSTE-based spiral design, we concluded that better performance could be achieved by reducing the length of the TE legs, while keeping the stretching capabilities to get the benefit from a higher temperature gradient. The second design was based on the PI and polyester paper with shorter leg's length, and single spiral. It featured a simpler fabrication process and displayed lower resistance, hence increasing the maximum output power. Moreover, the polyester paper-based TEG generated almost twice the power when stretched. Foldable paper-based TEGs were also demonstrated using two types of papers, fiber-based and polyester-based. Additionally, it was observed that the polyester-based paper produced almost three times more power than the standard paper. These papers-based sensors can be folded and unfolded, thus reducing or increasing the device size and making possible to adjust the power density. Lastly, we also studied how we can improve the mechanical stretchability performance by introducing compound structures, e.g. combining serpentine with spirals. To conclude, we believe our simple integration approach represent an important step towards wearable technologies with self-powering capabilities, and it offers an interesting and versatile methodology, which can be easily extrapolated to new materials and technologies for a greater variety of applications.

\section{Acknowledgment}

The authors would like to acknowledge the support provided by the Deanship of Scientific Research (DSR) at King Fahd University of Petroleum \& Minerals (KFUPM) for funding part of the presented work through project No. IN161020.

\section{REFERENCES}

[1] Choi, S., Lee, H., Ghaffari, R., Hyeon, T. and Kim, D. H., "Recent Advances in Flexible and Stretchable BioElectronic Devices Integrated with Nanomaterials," Adv. Mater. 28(22), 4203-4218 (2016).

[2] Rus, D. and Tolley, M. T., "Design, fabrication and control of soft robots," Nature 521(7553), 467-475 (2015).

[3] Hussain, A. M. and Hussain, M. M., "CMOS-Technology-Enabled Flexible and Stretchable Electronics for Internet of Everything Applications," Adv. Mater. 28(22), 4219-4249 (2016).

[4] Khan, Y., Ostfeld, A. E., Lochner, C. M., Pierre, A. and Arias, A. C., "Monitoring of Vital Signs with Flexible and Wearable Medical Devices," Adv. Mater. 28(22), 4373-4395 (2016).

[5] Cheng, M. Y., Tsao, C. M., Lai, Y. Z. and Yang, Y. J., "The development of a highly twistable tactile sensing array with stretchable helical electrodes," Sensors Actuators A Phys. 166(2), 226-233 (2011).

[6] Hwang, E. S., Seo, J. H. and Kim, Y. J., "A polymer-based flexible tactile sensor for both normal and shear load detections and its application for robotics," J. Microelectromechanical Syst. 16(3), 556-563 (2007). 
[7] Wang, C., Hwang, D., Yu, Z., Takei, K., Park, J., Chen, T., Ma, B. and Javey, a., "User-interactive electronic skin for instantaneous pressure visualization," Nat Mater 12(10), 899-904 (2013).

[8] Ostfeld, A. E., Gaikwad, A. M., Khan, Y. and Arias, A. C., "High-performance flexible energy storage and harvesting system for wearable electronics," Sci. Rep. 6, 26122 (2016).

[9] Kim, D.-H., Lu, N., Huang, Y. and Rogers, J. a., "Materials for stretchable electronics in bioinspired and biointegrated devices," MRS Bull. 37(3), 226-235 (2012).

[10] Rogers, J. A., Someya, T. and Huang, Y., "Materials and Mechanics for Stretchable Electronics," Science 327(5973), 1603-1607 (2010).

[11] Escabi, M. A., Read, H. L., Viventi, J., Kim, D.-H., Higgins, N. C., Storace, D. A., Liu, A. S. K., Gifford, A. M., Burke, J. F., Campisi, M., Kim, Y.-S., Avrin, A. E., Spiegel Jan, V. d., Huang, Y., Li, M., Wu, J., Rogers, J. A., Litt, B. and Cohen, Y. E., "A high-density, high-channel count, multiplexed ECoG array for auditory-cortex recordings," J. Neurophysiol. 112(6), 1566-1583 (2014).

[12] Harris, K. D., Elias, A. L. and Chung, H. J., "Flexible electronics under strain: a review of mechanical characterization and durability enhancement strategies," J. Mater. Sci. 51(6), 2771-2805 (2016).

[13] Yao, S. and Zhu, Y., "Nanomaterial-enabled stretchable conductors: Strategies, materials and devices," Adv. Mater. 27(9), 1480-1511 (2015).

[14] Park, S., Vosguerichian, M. and Bao, Z., "A review of fabrication and applications of carbon nanotube filmbased flexible electronics," Nanoscale 5(5), 1727-1752 (2013).

[15] Kim, D. H., Xiao, J., Song, J., Huang, Y. and Rogers, J. A., "Stretchable, curvilinear electronics based on inorganic materials," Adv. Mater. 22(19), 2108-2124 (2010).

[16] Sun, Y., Kumar, V., Adesida, I. and Rogers, J. A., "Buckled and wavy ribbons of GaAs for high-performance electronics on elastomeric substrates," Adv. Mater. 18(21), 2857-2862 (2006).

[17] Jones, J., Lacour, S. P., Wagner, S. and Suo, Z., "Stretchable wavy metal interconnects," J. Vac. Sci. Technol. A Vacuum Surfaces Film 22(2004), 1723 (2004).

[18] Li, T., Huang, Z., Suo, Z., Lacour, S. P. and Wagner, S., "Stretchability of thin metal films on elastomer substrates," Appl. Phys. Lett. 85(16), 3435-3437 (2004).

[19] Huang, K., Dinyari, R., Lanzara, G., Jong, Y. K., Feng, J., Vancura, C., Chang, F. K. and Peumans, P., “An approach to cost-effective, robust, large-area electronics using monolithic silicon," Tech. Dig. - Int. Electron Devices Meet. IEDM, 217-220 (2007).

[20] Rojas, J. P., Arevalo, A., Foulds, I. G. and Hussain, M. M., "Design and characterization of ultra-stretchable monolithic silicon fabric,” Appl. Phys. Lett. 105(15), 154101 (2014).

[21] Park, S. Il, Ahn, J. H., Feng, X., Wang, S., Huang, Y. and Rogers, J. A., "Theoretical and experimental studies of bending of inorganic electronic materials on plastic substrates," Adv. Funct. Mater. 18(18), 2673-2684 (2008).

[22] Kim, D. H., Xiao, J. L., Song, J. Z., Huang, Y. G. and Rogers, J. A., "Stretchable, curvilinear electronics based on inorganic materials," Adv. Mater. 22, 2108-2124 (2010).

[23] Rehman, M. U. and Rojas, J. P., "Optimization of compound serpentine-spiral structure for ultra-stretchable electronics,” Extrem. Mech. Lett. 15, 44-50 (2017).

[24] Qaiser, N., Khan, S. M., Nour, M., Rehman, M. U., Rojas, J. P. and Hussain, M. M., "Mechanical response of spiral interconnect arrays for highly stretchable electronics," Appl. Phys. Lett. 111(21), 214102 (2017).

[25] Rojas, J. P., Singh, D., Conchouso, D., Arevalo, A., Foulds, I. G. and Hussain, M. M., "Stretchable Helical Architecture Inorganic-Organic Hetero Thermoelectric Generator," Nano Energy 30, 691-699 (2016).

[26] Zhang, J., Liu, H. J., Cheng, L., Wei, J., Liang, J. H., Fan, D. D., Shi, J., Tang, X. F. and Zhang, Q. J., "Phosphorene nanoribbon as a promising candidate for thermoelectric applications," Sci. Rep. 4, 6452 (2014).

[27] Arab, A. and Li, Q., "Anisotropic thermoelectric behavior in armchair and zigzag mono-and fewlayer MoS 2 in 
thermoelectric generator applications," Sci. Rep. 5, 13706 (2015).

[28] Zhang, G. and Zhang, Y. W., "Strain effects on thermoelectric properties of two-dimensional materials," Mech. Mater. 91, 382-398 (2015).

[29] Rowe, D. M., “Thermoelectrics, an environmentally-friendly source of electrical power," Renew. Energy 16(1), 1251-1256 (1999).

[30] Zheng, X. F., Liu, C. X., Yan, Y. Y. and Wang, Q., “A review of thermoelectrics research - Recent developments and potentials for sustainable and renewable energy applications," Renew. Sustain. Energy Rev. 32, 486-503 (2014).

[31] Kim, S. J., We, J. H. and Cho, B. J., “A wearable thermoelectric generator fabricated on a glass fabric," Energy Environ. Sci. 7(6), 1959 (2014).

[32] Leonov, V. and Vullers, R. J. M., "Wearable electronics self-powered by using human body heat: The state of the art and the perspective," J. Renew. Sustain. Energy 1(6), 62701 (2009).

[33] Kim, M. K., Kim, M. S., Lee, S., Kim, C. and Kim, Y. J., "Wearable thermoelectric generator for harvesting human body heat energy," Smart Mater. Struct. 23(10), 105002 (2014).

[34] Du, Y., Cai, K., Chen, S., Wang, H., Shen, S. Z., Donelson, R. and Lin, T., "Thermoelectric fabrics: Toward power generating clothing," Sci. Rep. 5, 6411 (2015).

[35] Gao, C. and Chen, G., "Conducting polymer/carbon particle thermoelectric composites: Emerging green energy materials," Compos. Sci. Technol. 124, 52-70 (2016).

[36] Suemori, K., Hoshino, S. and Kamata, T., "Flexible and lightweight thermoelectric generators composed of carbon nanotube-polystyrene composites printed on film substrate," Appl. Phys. Lett. 103(15), 153902 (2013).

[37] Jiang, Q., Liu, C., Song, H., Xu, J., Mo, D., Shi, H., Wang, Z., Jiang, F., Lu, B. and Zhu, Z., "Free-standing PEDOT: PSS film as electrode for the electrodeposition of bismuth telluride and its thermoelectric performance,” Int. J. Electrochem. Sci. 9(12), 7540-7551 (2014).

[38] Zhang, Q., Sun, Y., Xu, W. and Zhu, D., "Organic Thermoelectric Materials: Emerging Green Energy Materials Converting Heat to Electricity Directly and Efficiently,” Adv. Mater. 26(40), 6829-6851 (2014).

[39] Aranguren, P., Roch, A., Stepien, L., Abt, M., Von Lukowicz, M., Dani, I. and Astrain, D., "Optimized design for flexible polymer thermoelectric generators," Appl. Therm. Eng. 102, 402-411 (2016).

[40] Ghafouri, N., Kim, H., Atashbar, M. Z. and Najafi, K., "A micro thermoelectric energy scavenger for a hybrid insect," Proc. IEEE Sensors, 1249-1252 (2008).

[41] Arevalo, A., Byas, E., Conchouso, D., Castro, D., Ilyas, S. and Foulds, I. G., “A versatile multi-user polyimide surface micromachinning process for MEMS applications," 2015 IEEE 10th Int. Conf. Nano/Micro Eng. Mol. Syst. NEMS, 561-565 (2015).

[42] Castro, D., Diaz, M., Arevalo, A., Conchouso, D. and Foulds, I. G., "Out-of-plane buckled cantilever microstructures with adjustable angular positions using thermal bimorph actuation for transducer applications," Micro Nano Lett. 10(10), 545-549 (2015).

[43] Conchouso, D., Arevalo, A., Castro, D. and Foulds, I. G., "Out-of-plane platforms with bi-directional thermal bimorph actuation for transducer applications," 2015 IEEE 10th Int. Conf. Nano/Micro Eng. Mol. Syst. NEMS, 608-612 (2015).

[44] Rojas, J. P., Conchouso, D., Arevalo, A., Singh, D., Foulds, I. G. and Hussain, M. M., "Paper-based origami flexible and foldable thermoelectric nanogenerator," Nano Energy 31, 296-301 (2017).

[45] Lamoureux, A., Lee, K., Shlian, M., Forrest, S. R. and Shtein, M., "Dynamic kirigami structures for integrated solar tracking," Nat. Commun. 6, 8092 (2015).

[46] Song, Z., Ma, T., Tang, R., Cheng, Q., Wang, X., Krishnaraju, D., Panat, R., Chan, C. K., Yu, H. and Jiang, H., "Origami lithium-ion batteries," Nat. Commun. 5, 3140 (2014). 
[47] Wang, Z. L., “Triboelectric nanogenerators as new energy technology for self-powered systems and as active mechanical and chemical sensors," ACS Nano 7(11), 9533-9557 (2013).

[48] Oh, J. Y., Lee, J. H., Han, S. W., Chae, S. S., Bae, E. J., Kang, Y. H., Choi, W. J., Cho, S. Y., Lee, J.-O., Baik, H. K. and Lee, T. Il., "Chemically exfoliated transition metal dichalcogenide nanosheet-based wearable thermoelectric generators,” Energy Environ. Sci. 9(5), 1696-1705 (2016).

[49] Wang, S., Huang, Y. and Rogers, J. A., "Mechanical Designs for Inorganic Stretchable Circuits in Soft Electronics," IEEE Trans. Components Packag. Manuf. Technol. 5(9), 1201-1218 (2015).

[50] Fan, J. a, Yeo, W.-H., Su, Y., Hattori, Y., Lee, W., Jung, S.-Y., Zhang, Y., Liu, Z., Cheng, H., Falgout, L., Bajema, M., Coleman, T., Gregoire, D., Larsen, R. J., Huang, Y. and Rogers, J. a., "Fractal design concepts for stretchable electronics.," Nat. Commun. 5, 3266 (2014).

[51] Xu, S., Yan, Z., Jang, K.-I., Huang, W., Fu, H., Kim, J., Wei, Z., Flavin, M., McCracken, J., Wang, R., Badea, A., Liu, Y., Xiao, D., Zhou, G., Lee, J., Chung, H. U., Cheng, H., Ren, W., Banks, A., et al., “Assembly of micro/nanomaterials into complex, three-dimensional architectures by compressive buckling," Science 347(6218), 154-159 (2015). 